\title{
COMPACTIFICATIONS OF DIMENSION ZERO
}

\section{J. HEIDER ${ }^{1}$}

1. Introduction. A c.r. space $X$ is here understood to be a completely regular $T_{1}$ space. A continuous function on $X$ is understood to be real-valued, and the symbol $C(X)$ denotes the collection of all such functions. For each element $f$ of $C(X)$, let $N(f)=[x: f(x)<0]$. Consider the following requirements with respect of the set $N(f)$ :

(I) $N(f)$ is closed (and open);

(II) $\bar{N}(f)$ is open (and closed);

(III) $N(f)$ is a countable union of open-and-closed (cloven) sets.

The c.r. spaces, in which each set $N(f)$ satisfies (I), are the $P$ spaces of $[2 ; 3]$ and enjoy many interesting alternate characterizations. The c.r. spaces, in which each set $\bar{N}(f)$ satisfies (II), are characterized as the c.r. spaces $X$ for which the lattice $C(X)$ is conditionally $\sigma$-complete, or, equivalently, for which the Stone-Čech compactification space $\beta X$ is the Boolean representation space of a $\sigma$-complete Boolean algebra $[3 ; 7]$.

Requirement (III) drastically weakens (I). Yet, the first purpose of this note is to show that (III) characterizes an important class of c.r. spaces, viz., those c.r. spaces $X$ for which $\beta X$ is of dimension zero, in the sense of possessing a base of cloven sets. From this it will follow that $(\mathrm{I}) \Rightarrow(\mathrm{II}) \Rightarrow$ (III).

If $X$ is of dimension zero, attention focuses on the field of cloven subsets of $X$. If, moreover, $\beta X$ is of dimension zero, it is the Boolean representation space of this field of sets, viewed as partially ordered by the inclusion relation. These are set-theoretic considerations. Opposed to this, requirement (III) involves the consideration of continuous functions. In addition to (III), there are two established $[3 ; 5]$ characterizations of the c.r. spaces $X$ for which $\beta X$ is of dimension zero: (i) for each pair $A$ and $B$ of separated sets in $X$, there is a cloven set in $X$ containing $A$ and disjoint from $B$; (ii) each finite normal open covering of $X$ possesses a refinement which is a finite partition of $X$ into cloven sets. Condition (i), in the concept of separated sets, refers to continuous functions. The concept of a normal covering, employed in condition (ii), is difficult of expression in any form and is, perhaps, best expressed in terms of continuous functions [4]. The second purpose of this note is, therefore, to replace condi-

Presented to the Society, November 28, 1958; received by the editors July 16, 1958.

1 This research was supported by the National Science Foundation. 
tions (i) and (ii) and likewise requirement (III) by an equivalent condition based exclusively on set-theoretic concepts. This is accomplished for all c.r. spaces, but with the most notable simplicity for normal spaces.

For c.r. spaces $X$ with $\beta X$ of dimension zero, the preceding emphasis on set-theoretic techniques should extend to the consideration of continuous functions. The concluding section of this note describes a countable, purely set-theoretic technique for construction of all continuous functions pertaining to a c.r. space $X$ with $\beta X$ of dimension zero.

2. Compactifications of dimension zero. Let $X$ be a c.r. space, and let $f$ be an element of $C(X)$ with finite bounds $a$ and $b$. Then, in terms of Stone's spectral representation of continuous functions [7], to each real number $r$ there is assigned an open subset $E_{f}(r)$ of $X$ such that:

(1) $E_{f}(r)=\phi$ for all $r \leqq a$,

(2) $E_{f}(r)=U_{r^{\prime}<r} E_{f}\left(r^{\prime}\right)$ for all $r$,

(3) $\bar{E}_{f}\left(r^{\prime}\right) \subseteq E_{f}(r)$ for $r^{\prime}<r$,

(4) $E_{f}(r)=X$ for $r>b$.

Conversely, any family $[E(r)]_{r \in R}$ of open subsets of $X$, satisfying conditions (1)-(4) for some pair $a \leqq b$ of real numbers, determines uniquely a bounded continuous function $f$ on $X$ with $[x: f(x)<r]$ $=E(r)$.

The significance of requirement (III) of the introduction is now partly revealed.

Theorem 2.1. If a c.r. space $X$ is such that $\beta X$ is of dimension zero, then each spectral set $E_{f}(r)$, and thus the set $N(f)$, of each element $f$ of $C(X)$ is a countable union of cloven subsets of $X$.

Proof. Let $f$ be a bounded continuous function on $X$, and let $\bar{f}$ be its unique extension over $\beta X$, the latter being assumed of dimension zero. For arbitrary real numbers $r_{1}<r_{2}, E_{\bar{f}}\left(r_{1}\right) \subseteq\left[p \in \beta X: \bar{f}(p) \leqq r_{1}\right]$ $\subseteq E_{\bar{j}}\left(r_{2}\right)$. With $\beta X$ compact and of dimension zero, a cloven subset $G$ of $\beta X$ may be chosen with $\left[p \in \beta X: \bar{f}(p) \leqq r_{1}\right] \subseteq G \subseteq E_{\bar{f}}\left(r_{2}\right)$. Then $E_{f}\left(r_{1}\right) \subseteq 0 \subseteq E_{f}\left(r_{2}\right)$, where 0 is the cloven residue in $X$ of the cloven subset $G$ of $\beta X$.

Finally, with $\left\{r_{n}\right\}$ a monotone increasing sequence of numbers approaching the number $r$ as a limit, from $E_{f}(r)=U_{r^{\prime}<r} E_{f}\left(r^{\prime}\right)$ $=\bigcup_{n=1}^{\infty} E_{f}\left(r_{n}\right)$, one concludes that $E_{f}(r)$ is a countable union of cloven subsets of $X$.

Assume, now, that the c.r. space $X$ is such that each set $N(f)$, for $f$ in $C(X)$, is a countable union of cloven subsets of $X$. Note that $N(-f)=P(f)=[x: f(x)>0]$ and $E_{f}(r)=N(f-r)$. With $r_{1}<r_{2}$, for any 
element $f$ of $C(X)$, the set $\left[x: f(x) \leqq r_{1}\right]$ is a countable intersection of cloven subsets and is contained in the countable union of cloven sets $\left[x: f(x)<r_{2}\right]$. The following lemma is now useful:

Lemma 2.2. If $\left\{0_{n}\right\}$ and $\left\{G_{n}\right\}$ are two sequences of cloven subsets of a topological space $X$, with $\cap_{n=1}^{\infty} 0_{n} \subseteq \bigcup_{n=1}^{\infty} G_{n}$, then there exists a cloven subset $L$ of $X$ with $\bigcap_{n=1}^{\infty} 0_{n} \subseteq L \subseteq \bigcup_{1=n}^{\infty} G_{n}$.

PROOF. Form a sequence $\left\{H_{n}\right\}$ of cloven sets, with $H_{2 n-1}=G_{n}$ and $H_{2 n}=\tilde{0}_{n}$, where $\tilde{0}_{n}$ denotes the cloven complement in $X$ of $0_{n}$. Each point of $X$ is in some set $H_{n}$. Next form $\left\{H_{n}^{*}\right\}$ with $H_{n}^{*}=H_{n}-U_{1 \leq i<n} H_{i}$. The sets $H_{n}^{*}$ are cloven, pairwise disjoint and cover $X$. Finally, let $L$ denote the point-set union of all $H_{n}^{*}$ containing points of the set $\bigcap_{n=1}^{\infty} 0_{n}$, while $M$ denotes the union of the remaining $H_{n}^{*}$. Then $L$ and $M$, as disjoint unions of open sets with $L \cup M=X$, are seen to be cloven subsets of $X$. Finally, $\bigcap_{n=1}^{\infty} 0_{n} \subseteq L \subseteq \bigcup_{n=1}^{\infty} G_{n}$ as desired.

Let $X$ continue to denote a c.r. space for which all sets $N(f)$, with $f$ in $C(X)$, are countable unions of cloven sets. It is clear that the cloven sets then constitute a base for the topology on $X$. These cloven sets, as ordered by the inclusion relation, form a Boolean algebra. Let $\eta X$ denote the Boolean representation space of this algebra. The points of $\eta X$ will here be regarded as prime dual ideals of cloven sets of $X$, i.e., proper subsets of the collection of all cloven sets, closed under finite intersections of their elements, and maximal with respect to these properties. Each point of $X$ determines, uniquely, such a prime dual ideal, and $X$ is seen to be imbedded as a dense subspace of $\eta X$.

In general, for any c.r. space $X$ of dimension zero, the space $\eta X$ serves as a maximal compact space of dimension zero, containing $X$ as a dense subspace [1]. However, under the present assumptions, $\eta X=\beta X$, the Stone-Cech compactification space of $X$. Thus, as appears from the preceding discussion, for any (bounded) element $f$ of $C(X)$ and any pair of numbers $r_{1}<r_{2}$, there exists a cloven subset 0 of $X$ with $E_{f}\left(r_{1}\right) \subseteq 0 \subseteq E_{f}\left(r_{2}\right)$. However, each prime dual ideal (point) of $\eta X$ contains either this cloven set 0 or its complement. Then, using only the fact that a sequence of closed, nested intervals of the real line, of widths approaching zero, determines a unique real number, for each prime dual ideal (point) $\alpha$ of $\eta X$ and for each bounded element $f$ of $C(X)$, it is possible to assign a real number $\bar{f}(\alpha)$ such that, for each $\epsilon>0$, there is a cloven set 0 in $\alpha$ with $|\bar{f}(\alpha)-f(x)|<\epsilon$ for each point $x$ of $X$ in 0 . Clearly the function $\bar{f}$ thus defined on $\eta X$ is a continuous extension of the given bounded element $f$ of $C(X)$.

In view of this discussion and of Theorem 2.1, our first contention is now established. 
THEOREM 2.3. The c.r. spaces $X$ for which $\beta X$ is of dimension zero are the spaces $X$ for which each set $N(f), f$ in $C(X)$, is a countable union of cloven sets.

3. A set-theoretic characterization. The purpose of this section is to reduce the characterization of the c.r. spaces $X$ for which $\beta X$ is of dimension zero to the simplest possible set-theoretic terms. To this end, a definition and theorem of recent origin [6] will be of service.

Definition. Let $\mathscr{T}$ be any family of open subsets of a c.r. space $X$. Then $\mathfrak{T}$ will be called a completely regular system of open sets if, for each element $M$ of $\mathscr{T}$, there are sequences $\left\{M_{n}^{\prime}\right\}$ and $\left\{M_{n}^{\prime \prime}\right\}$ of elements of $\Re$ with $M_{n}^{\prime} \subseteq \tilde{M}_{n}^{\prime \prime} \subseteq M$ and $M=\cup_{n=1}^{\infty} M_{n}^{\prime}$. (Here $\tilde{M}_{n}^{\prime \prime}$ denotes the complement in $X$ of the set $M_{n}^{\prime \prime}$.)

For any element $f$ of $C(X)$, with $X$ a c.r. space, the system of all subsets $f^{-1}(0)$ of $X$, with 0 an open subset of the real line, is clearly a completely regular system of open subsets of $X$. However, even more can be asserted.

THEOREM. Every element of any completely regular system of open sets of a c.r. space $X$ has the form $f^{-1}(0)$ for some element $f$ of $C(X)$ and some open subset 0 of the real line.

The proof of this theorem, as it appears in [6], is an interpretation of involved properties of certain abstract systems. However, the theorem itself has a simple application to our present task.

Theorem 3.1. The c.r. spaces $X$ for which $\beta X$ is of dimension zero are the spaces in which the system of all countable unions of cloven sets includes every completely regular system of open sets.

To establish this theorem, it needs only to be noted that sets of the type $f^{-1}(0)$ include all spectral sets, while each set of the type $f^{-1}(0)$ is a countable union of finite intersections of spectral sets $E_{f}(r)$ and $E_{g}(r)$, where $g=-f$ and $f$ is an element of $C(X)$. For any c.r. space $X$, the system of all countable unions of cloven sets obviously constitutes a completely regular system of open sets. Thus, the theorem simply requires that this particular system be the greatest such completely regular system of open sets.

If $\beta X$ is to be of dimension zero, the topology of $X$ must be based on the field $\mathcal{F}$ of cloven subsets of $X$. This field must be reduced, in the sense that distinct points of $X$ are contained in complementary elements of $\mathcal{F}$, and it must be union-intersection closed, in the sense that any subset of $X$ which is both an intersection and a union of elements of $\mathcal{F}$ is itself an element of $\mathcal{F}$. 
Now let $\mathcal{F}$ be such a reduced, union-intersection closed field of subsets of a set $X$. The symbol 0 , with or without subscript, will be reserved to denote elements of $\mathcal{F}$. Let $X(\mathcal{F})$ indicate the c.r. space obtained by using the elements of $\mathcal{F}$ as a base for open sets in $X$. The symbol $\cup 0_{\mu}$ will denote a general open set of $X(\mathcal{F})$, while $\bigcup_{r} 0_{\mu}$ will indicate an open set contained in a completely regular system of open sets, and $\bigcap_{r} 0_{\nu}$ will indicate the complement in $X$ of such a set. Finally, let $\eta X(\mathcal{F})$ represent the Boolean representation space of $\mathfrak{F}$. The basic question concerns set-theoretic conditions under which $\eta X(\mathcal{F})=\beta X(F)$.

TheOREM 3.2. The condition $\left\{\bigcap_{r} 0_{\nu} \subseteq \cup_{r} 0_{\mu} \Rightarrow \exists 0\right.$ with $\left.\bigcap_{r} 0_{\nu} \subseteq 0 \subseteq U_{r} 0_{\mu}\right\}$ is equivalent to the condition that $\eta X(\mathcal{F})=\beta X(\mathfrak{F})$.

Theorem 3.3. The condition $\left\{\cap 0_{\nu} \subseteq \mathrm{U}_{\mu} \Rightarrow \exists 0\right.$ with $\left.\cap 0_{\nu} \subseteq 0 \subseteq \mathrm{U}_{\mu}\right\}$ is equivalent to the double condition that $X(F)$ be normal and $\eta X(F)$ $=\beta X(\mathfrak{F})$.

As regards Theorem 3.2, if $\eta X(\mathcal{F})=\beta X(F)$, then, by Theorem 3.1, each $\bigcap_{r} 0_{\nu}$ is a countable intersection and each $U_{r} 0_{\mu}$ is a countable union, and Lemma 2.2 applies. Conversely, if $\bigcap_{r} 0_{\nu} \subseteq U_{r} 0_{\mu}$ always allows $\bigcap_{r} 0_{\nu} \subseteq 0 \subseteq U_{r} 0_{\mu}$, then each $U_{r} 0_{\mu}$, as a member of a completely regular system of open sets, is seen to be a countable union of cloven sets, and Theorem 3.1 applies.

As regards Theorem 3.3, if $\cap 0_{\nu} \subseteq \cup 0_{\mu}$ always allows $\cap 0_{\nu} \subseteq 0 \subseteq \cup 0_{\mu}$, then $X(F)$ is obviously normal and, by Theorem $3.2, \eta X(F)=\beta X(F)$. Conversely, with $\beta X(F)=\eta X(F)$ and thus of dimension zero, from the normality of $X(F)$ it is clear that $\cap_{\nu_{\nu}} \subseteq \mathrm{U} 0_{\mu}$ always allows $\bigcap_{\nu_{\nu}} \subseteq 0$ $\subseteq \mathrm{U}_{\mu}$.

As an application of Theorem 3.3, with $X, F, X(F)$ and $\eta X(F)$ as described above, certain cases wherein $X(F)$ is normal and $\eta X(F)$ $=\beta X(F)$ may be noted:

(a) $X(\mathcal{F})$ possesses a countable base of open sets;

(b) each $U 0_{\mu}$ may be represented as a countable union of cloven sets;

(c) $X(F)$ has the Lindelöf property;

(d) each binary covering of $X(\mathcal{F})$ by open sets may be refined by a partition of $X$ into cloven sets.

As an application of Theorem 3.2, the following case is of some importance. Let $\mathfrak{F}$ continue to denote a reduced, union-intersection closed field of subsets of a set $X$. However, now also assume that $F$ is a $\sigma$-field, in the sense that any countable union of elements of $\mathcal{F}$ is again an element of $\mathcal{F}$. Let $\mathfrak{T}$ denote a completely regular system of 
open subsets of $X(\mathcal{F})$, and let $M$ be an element of $\mathfrak{T}$. Then, by definition, there exists a sequence $\left\{{M_{n}^{\prime \prime}}^{\prime \prime}\right\}$ of elements of $\mathfrak{T}$ with $M$ $=\bigcup_{n=1}^{\infty} \tilde{M}_{n}^{\prime \prime}$. Each element $\tilde{M}_{n}^{\prime \prime}$ is of the form $\tilde{M}_{n}^{\prime \prime}=\bigcap_{\nu \in I_{n}} 0_{n, \nu}$ where each $0_{n, \nu}$ is an element of $\mathcal{F}$ and each $I_{n}$ is an index set. Then

$$
M=\bigcup_{n=1}^{\infty}\left[\bigcap_{\nu \in I_{n}} 0_{n, \nu}\right]=\bigcap_{h \in H}\left[\bigcup_{n=1}^{\infty} 0_{n, h(n)}\right]
$$

where $H$ is the collection of all functions $h$ on the positive integers with $h(n)$ in $I_{n}$. However, each $\bigcup_{n=1}^{\infty} 0_{n, h(n)}$, as a countable union of elements of $\mathcal{F}$, is an element $0_{h}$ of $\mathcal{F}$. Hence $M=\bigcap_{h \in H} 0_{h}$, and $M$ is seen to be a cloven set in $X(\mathcal{F})$ and thus an element of $\mathcal{F}$. The c.r. spaces $X(F)$ constructed with elements of a reduced, union-intersection closed, $\sigma$-field $\mathcal{F}$ of subsets of $X$ as a base for open sets are identical with the $P$ spaces of $[2 ; 3]$, i.e., the c.r. spaces $X$ for which each set $N(f), f$ in $C(X)$, is closed (and open).

4. The construction of continuous functions. Let the c.r. space $X$ be such that $\beta X$ is of dimension zero. Let $f$ be an element of $C(X)$ and, for the sake of simplicity, assume that $f$ is bounded with $0 \leqq f(x)$ $\leqq 1$ throughout $X$. As indicated in the proof of Theorem 2.1, for each pair $r_{1}<r_{2}$ of real numbers, there is a cloven subset 0 of $X$ with $E_{f}\left(r_{1}\right) \subseteq 0 \subseteq E_{f}\left(r_{2}\right)$. Now let $r_{2}=p / 2^{n}$ where $p$ is an odd positive integer $1 \leqq p<2^{n}$ and let $r_{1}=(2 p-1) / 2^{n+1}$. Let $\left[p / 2^{n}\right]$ symbolize a cloven set such that $E_{f}\left(2 p-1 / 2^{n+1}\right) \subseteq\left[p / 2^{n}\right] \subseteq E_{f}\left(p / 2^{n}\right)$.

As $r_{2}=p / 2^{n}$ exhausts the sets $1 / 2 ; 1 / 4,3 / 4 ; 1 / 8, \cdots$, an array of cloven sets is formed:

$$
\begin{aligned}
& {[1 / 2]} \\
& {[1 / 4] \subseteq[1 / 2] \subseteq[3 / 4]} \\
& [1 / 8] \subseteq[1 / 4] \subseteq[3 / 8] ;[1 / 2]\} \subseteq[5 / 8] \subseteq[3 / 4] \subseteq[7 / 8] \\
& [1 / 16] \subseteq[1 / 8] \subseteq[3 / 16] ;[1 / 4]\} \subseteq[5 / 16] \subseteq[3 / 8] \subseteq[7 / 16] \\
& [1 / 2]\} \subseteq[9 / 16] \subseteq \ldots
\end{aligned}
$$

Here $\subseteq$ denotes set inclusion. The symbol $\} \subseteq\left[p / 2^{n}\right]$ indicates that the set $\left[p / 2^{n}\right]$ contains every set to the left of the bracket in the row under consideration. Finally the presence of the semi-colon, rather than the inclusion symbol, indicates that no inclusion relation is asserted in regard to the sets immediately adjacent to the semicolon. 
From this array, the spectral sets $E_{f}(r)$ of the element $f$ of $C(X)$, and thus the element $f$ itself, can be recovered. Thus, for $0<r \leqq 1$, $E_{f}(r)=U_{0<p / 2^{n}<r}\left[p / 2^{n}\right]$, while $E_{f}(r)=\phi$ for $r \leqq 0$, and $E_{f}(r)=X$ for $r>1$.

Now let $\left\{0_{n}\right\}$ denote any sequence of cloven subsets of $X$. Let $[1 / 2]=0_{1}$. Let $[1 / 4]=0_{2} \cap[1 / 2]$ and $[3 / 4]=0_{3} \cup[1 / 2]$. Let $[1 / 8]$ $=0_{4} \cap[1 / 4],[3 / 8]=\left(0_{5} \cup[1 / 4]\right) \cap[3 / 4],[5 / 8]=\left(0_{6} \cup[3 / 8] \cup[1 / 2]\right)$ $\cap[3 / 4]$ and $[7 / 8]=0_{7} \cup[3 / 4]$. Continuing in this manner, through the exclusive use of finite set unions and intersections, an array of cloven sets $\left[p / 2^{n}\right]$ is constructed enjoying the inclusion relations indicated above.

Now consider a quadruple

$$
\begin{aligned}
&\} \subseteq\left[(4 m+1) / 2^{n}\right] \subseteq\left[(4 m+2) / 2^{n}\right] \subseteq\left[(4 m+3) / 2^{n}\right] ; \\
& {\left.\left[(4 m+4) / 2^{n}\right]\right\} \subseteq . }
\end{aligned}
$$

An easy induction shows that, for $0<p / 2^{s} \leqq(4 m+1) / 2^{n},\left[p / 2^{s}\right]$ $\subseteq\left[(4 m+3) / 2^{n}\right]$. In fact, in the illustrative array derived above from a continuous function by the stated process, actually $\left[p / 2^{s}\right]$ $\subseteq\left[(4 m+2) / 2^{n}\right]$. An elementary, but difficult to describe, change in the procedure for constructing the $\left[p / 2^{n}\right]$ from a given sequence $\left\{0_{n}\right\}$ of cloven sets would accomplish the same effect, but the change is unnecessary.

With the array of $\left[p / 2^{n}\right]$ formed as above from the given $\left\{0_{n}\right\}$, let $E(r)=U_{0<p / 2^{n}<r}\left[p / 2^{n}\right]$ for $0<r \leqq 1$, while $E(r)=\phi$ for $r \leqq 0$ and $E(r)=X$ for $r>1$. For each pair $0 \leqq r_{1}<r_{2} \leqq 1$ of real numbers, it is clearly possible to find positive integers $m$ and $n$ such that $r_{1}<(4 m+1) / 2^{n}<(4 m+3) / 2^{n}<r_{2}$. Then $E\left(r_{1}\right) \subseteq\left[(4 m+3) / 2^{n}\right] \subseteq E\left(r_{2}\right)$ where $\left[(4 m+3) / 2^{n}\right]$ is a cloven set. From this it follows that the family $[E(r)]_{r \in R}$, thus constructed, is the spectral family of a (bounded) continuous function on $X$.

Since spectral families for unbounded continuous functions can be obtained from the spectral families of continuous functions $f$ with $0<f(x)<1$ throughout $X$, the stated procedure suffices to describe all elements of $C(X)$ when $\beta X$ is of dimension zero.

In conclusion, the following may be noted. Let $\mathcal{F}$ be a reduced, union-intersection closed field of subsets of a set $X$, and let $X(F)$ and $\eta X(F)$ be as described earlier. Then the bounded, continuous functions on $X(F)$ that can be extended over $\eta X(F)$ are precisely those bounded functions on $X(F)$ that are formed by the above countable, set-theoretic procedure, as applied to the subsets of $X$ in $F$. 


\section{BIBLIOGRAPHY}

1. B. Banaschewski, Über nulldimensionale Räume, Math. Nachr. vol. 13 (1955) pp. $129-140$.

2. L. Gillman and M. Henriksen, Concerning rings of continuous functions, Trans. Amer. Math. Soc. vol. 77 (1954) pp. 340-362.

3. - Rings of continuous functions in which every finitely generated ideal is principal, ibid. vol. 82 (1956) pp. 366-391.

4. M. Henriksen and J. Isbell, Local connectedness in the Stone-Cech compactification, Illinois J. Math. vol. 1 (1957) pp. 574-582.

5. J. Isbell, Zero-dimensional spaces, Tôhoku Math. J. vol. 7 (1955) pp. 1-8.

6. J. Kerstan, Eine Charakterisierung der vollständig regulären Räume, Math. Nachr. vol. 17 (1958) pp. 27-46.

7. M. Stone, Boundedness properties in function lattices, Canad. J. Math. vol. 1 (1949) pp. 176-186.

INSTITUTE fOR AdVANCEd STUdy AND

MARQUETTE UNIVERSITY 